\title{
Violencia y salud en Colombia ${ }^{1}$
}

\author{
Saúl Franco Agudelo²
}

RESUMEN La violencia en Colombia parece incontenible. A las masacres y asesinatos colectivos, de una crueldad desconcertante, se suman los secuestros y desapariciones, el maltrato de niños y ancianos y las violaciones de adolescentes. Cada día, sin tregua, los colombianos son espectadores o víctimas de atracos callejeros y de discriminación racial, sexual y socioeconómica. Sin quererlo, se convierten en agentes de agresiones en el transporte urbano, el hogar, la escuela y el trabajo.

Colombia tiene las tasas de mortalidad por homicidio más altas del mundo. Independientemente de la enorme sobrecarga institucional que impone a los servicios de salud y medicina legal, la violencia constituye actualmente en Colombia el principal problema de salud pública. Para hacerle frente, el sector de la salud debe elaborar políticas y financiar acciones; crear nuevos procesos de formación de personal, poner en marcha procesos de educación pública, y dedicar mayor esfuerzo y creatividad al campo de la investigación, que hasta ahora ha dado respuestas importantes pero aún insuficientes.

Al reflexionar sobre la violencia, que es la sustitución de toda argumentación por la fuerza, es necesario ubicarla en el contexto de la vida y la salud. No se pretende solo racionalizarla, y menos aún sustituir la acción por la palabra o por las reflexiones, sino tratar de comprenderla a fondo con el objeto de buscarle alternativas.

En el presente artículo se analiza, con esta finalidad, el tema de la violencia en Colombia, principalmente desde el punto de vista de su efecto en la salud de los colombianos y de sus implicaciones para el sector sanitario. El autor expone sus puntos de vista con plena conciencia de su subjetividad y limitaciones.

Hasta mediados de los años setenta del presente siglo, en Colombia "La Violencia" tenía un significado causal y temporal preciso: el conjunto de actos de fuerza, generalmente atroces, cometidos al amparo de motivaciones predominantemente políticas y partidistas entre 1948 y 1964. Toda una extensa bibliografía, desde La violencia

\footnotetext{
1 Próximamente se publicará en esta revista una versión en inglés de este artículo.

2 Universidad Nacional de Colombia, Instituto de Estudios Políticos y Relaciones Internacionales, Santa Fe de Bogotá, Colombia. Dirección postal: Director, Corporación Salud y Desarrollo, Apartado Aéreo 57736, Santa Fe de Bogotá, Colombia. Correo electrónico: sfranco@gaitana.interred.net.co.
}

en Colombia (1), libro clásico que inició el estudio de la violencia en el país, hasta Once ensayos sobre La Violencia (2), se refiere a la misma unidad temática y cronológica, con obvias variaciones de enfoque, énfasis e interpretación.

El incremento de la violencia en todas sus formas al inicio de los años ochenta hizo pensar en una "nueva violencia", en abandonar la acepción temporal vigente y enfocar nuevas preguntas y problemas. Un grupo de consulta convocado por el gobierno nacional abordó el tema en 1987, intentando una especie de tipificación de la violencia y sugiriendo alternativas estructurales y sintomáticas. Para no caer en diferentes reduccionismos, en su documento decidieron definir la violencia como "todas aquellas actuaciones de individuos o grupos que ocasionen la muerte de otros o lesionen su integridad física o moral. En sentido muy general la violencia se puede ver como algo [sic] que impide la realización de los Derechos $\mathrm{Hu}$ manos, comenzando por el fundamental: el derecho a la vida" (3). Aparece entonces la violencia como actividad humana nociva que menoscaba la integridad y que limita los derechos. El documento citado constituye un hito en la definición del concepto y del problema de la violencia en el país. 
Desde su promulgación, la violencia en Colombia empieza a reconocerse como realidad polimorfa, multicausal, presente en todo el tejido social e individual.

En varios documentos anteriores $(4,5)$ he desarrollado algunas ideas sobre el perfil de ese complejo problema que llamamos violencia. En síntesis, se trata de la imposición de la fuerza, al servicio de determinado interés o conjunto de intereses, ejercida en condiciones de asimetría y con una dirección específica, con la consiguiente negación o limitación de uno o más de los derechos de sus víctimas. No es, como suele creerse, el producto de una determinación genética, del azar o de la carencia de lógica. Es una actividad humana consciente e inteligente, con una clara finalidad, que se origina en el desarrollo de las formas de relación entre humanos. Es, por lo tanto, cambiante e histórica. Se expresa en actos concretos pero requiere y supone determinados contextos, motivaciones, legalidades y escalas valorativas. Pero tampoco termina con los actos, ya que genera nuevos procesos y respuestas y produce alteraciones y consecuencias en los ámbitos individual y colectivo. Más que un proceso, es un conjunto de procesos. Tiene raíces, finalidades, consecuencias mediatas e inmediatas. Es un lenguaje sin palabras, material y simbólico. Cada acto violento deja víctimas, hiere, duele o mata y, al mismo tiempo, representa en lenguaje cifrado confrontaciones, luchas de poder, el surgimiento o la reafirmación de fuerzas y proyectos.

El Centro Latinoamericano de Estudios de Violencia y Salud, uno de los grupos que han venido examinando el tema en el Brasil, insiste en que la violencia se genera y desarrolla en la vida en sociedad, resaltando su especificidad histórica y la convergencia de problemas políticos, económicos, morales, legales, psicológicos, humanos e institucionales que representa, incluso en el plano individual. El mismo grupo ha subrayado que la violencia es una red en la cual no siempre somos víctimas, sino que con frecuencia nos convertimos en agresores (6).
En la última década la OPS se ha venido interesando en el tema y ha apoyado convocatorias para examinar la forma de enfrentar el problema en el sector de la salud. En algunos de sus documentos aún prima el énfasis en el aspecto conductual de la violencia (7) $\mathrm{y}$, por tanto, las propuestas de acción se concentran en el comportamiento individual. Obviamente, la OPS no tiene una posición monolítica al respecto. Producciones financiadas por ella o algunos de sus funcionarios temporeros han contribuido a ampliar el horizonte teórico, a explorar los aspectos políticos de la violencia y a aumentar la información disponible sobre el tema (8-10).

Finalmente, la presente reflexión acerca de los nexos entre la violencia y la salud se concentra en el caso de Colombia. La magnitud y tendencias que muestran los indicadores de algunas formas de violencia - el homicidio y el secuestro, por ejemplo- hacen que el país sea el mejor escenario mundial para explorar los nexos en cuestión. Aunque una caracterización de la actual realidad nacional va más allá de las posibilidades y objetivos de este documento, es esencial hacer algunos señalamientos mínimos. En primer lugar, es preciso reafirmar que la violencia no es un patrimonio exclusivo nacional, que Colombia no tiene las cifras máximas de la mayor parte de las formas de violencia y que no existen marcadores genéticos o niveles hormonales específicos que hagan de los colombianos un grupo humano particularmente violento. Irrefutablemente, existen algunos elementos culturales -unos explorados (11-14) y otros por explorar- y una compleja coyuntura nacional en la que convergen muchos elementos: las relaciones internacionales; la falta de equidad y la exclusión de ciertos grupos de la vida económica y política del país; el narcotráfico; algunas crisis y transiciones en la ética y en los valores; conflictos políticos y militares; la inoperancia de la justicia y el aumento de la impunidad (15-20). Cabe anotar también la importancia de la diversidad regional, cultural, étnica y política que consti- tuye la realidad global de Colombia. Las marcadas diferencias de estructura y dinámica familiar entre la costa y la región andina, por ejemplo, hacen que la violencia intrafamiliar tenga también perfiles e intensidades diferentes, como también los tienen la violencia urbana y la rural, la de las ciudades grandes y pequeñas, o la de las áreas en que el narcotráfico, la guerrilla o ambos han tenido una mayor o menor penetración. Un discurso sobre la violencia en Colombia tiene que dar cuenta de esas y otras diferenciaciones.

\section{EFECTO EN LA SALUD DE LOS COLOMBIANOS}

Resulta imposible elaborar y presentar un cuadro completo de la relación entre la violencia y la salud en Colombia. A la complejidad de la relación se suman las graves deficiencias de información. En Colombia, como en muchos otros países, hay problemas con los códigos e indicadores utilizados, los sucesos registrados y la cobertura y calidad de los sistemas de información sobre la violencia, tanto en términos generales como en el sector de la salud. Reconociendo estas limitaciones, destaco algunos aspectos del problema y algunos indicadores y sus tendencias para dar una idea de la magnitud del problema y de la urgencia con que hay que tomar decisiones y acciones.

\section{La epidemia de homicidios}

Hace tres décadas la violencia, entendida como el conjunto denominado "causas externas" en la nomenclatura empleada por el sector de la salud, ocupaba el noveno lugar entre las causas de muerte en Colombia. En los años setenta pasó a ocupar el cuarto lugar y desde los años ochenta se ha ubicado en el primero, tomando cada vez mayor ventaja en relación a las demás causas de muerte de los colombianos. Pero los verdaderos indicadores del incremento de la violencia en Colombia son el número y la tasa de 
homicidios. En la última década ha habido en el país más de 230000 homicidios, cifra que supera a los 200000 estimados en Colombia en los años cuarenta y cincuenta, durante el período de La Violencia. En 1994 los homicidios constituyeron $70 \%$ de todas las muertes violentas registradas en el país, según datos suministrados por el Instituto Nacional de Medicina Legal y Ciencias Forenses (INMLCF) (21). Según datos del Ministerio de Salud (cuadro 1), de 1987 a 1994 la tasa de homicidios aumentó de 36 a 127 por 100000 habitantes. Otras fuentes (figura 1) revelan tasas ligeramente distintas con una tendencia similar. En la actualidad, Colombia es el país con la tasa de homicidios más alta del mundo.

Aún más alarmante que el número total de homicidios es su distribución por sexo y grupos de edad. Los hombres jóvenes -y cada vez más jóvenes- son las víctimas más frecuentes de esta forma de violencia, de la que tampoco escapan las mujeres. Mientras que en la década pasada Medellín registró 18 veces más asesinatos de hombres que de mujeres (22), en 1994 registró solo 13 veces más (21). Aunque se mantuvo el predominio de hombres, la participación femenina mostró un incremento notable. Según el INMLCF, en 1994 el grupo de 15 a 24 años de edad fue víctima de $34,2 \%$ de todos los homicidios en el país, y el de 25 a 34 años de 33,3\%, lo cual indica que casi $70 \%$ de las víctimas de todos los homicidios tenían de 15 a 34 años de edad (21).

Como indica la figura 2, donde se presenta la distribución de homicidios en Colombia en 1994 por edad y sexo, es alarmante el número de niños que fueron víctimas de asesinatos. Al empezar la década de los noventa, el homicidio era la segunda causa de muerte en el grupo de 5 a 14 años (23). En los primeros 8 meses de 1995, solo en la ciudad de Medellín se notificaron 700 muertes violentas en menores de 18 años (24). En Itagüí, una de las 10 ciudades del país con el mayor número de necropsias en 1994, los homicidios fueron la principal forma de
CUADRO 1. Homicidios (por 100000 habitantes). Colombia, 1987 a 1994

\begin{tabular}{cc}
\hline Año & Homicidios \\
\hline 1987 & 36 \\
1988 & 53 \\
1989 & 68 \\
1990 & 73 \\
1991 & 81 \\
1992 & 86 \\
1993 & 106 \\
1994 & 127 \\
Total & 630 \\
\hline
\end{tabular}

Fuente: Datos tomados de la referencia 21.

muerte violenta en todos los grupos de edad a partir de los 5 años (21). Ese mismo año, las tres principales ciudades colombianas — Santa Fe de Bogotá, Cali y Medellín-, que abarcan la tercera parte de la población del país, tuvieron $50 \%$ de todos los homicidios registrados. Este dato respalda la hipótesis de la urbanización de la violencia que actualmente sacude al país, fenómeno que la distingue de la violencia clásica ya mencionada (25). Según datos de la Policía Nacional, en 1990 $85 \%$ de los delitos contra el patrimonio económico y $78 \%$ de los delitos contra
FIGURA 1. Tasas de mortalidad (por 100000 habitantes) por homicidios, accidentes de tránsito y suicidios. Colombia, 1984 a 1990

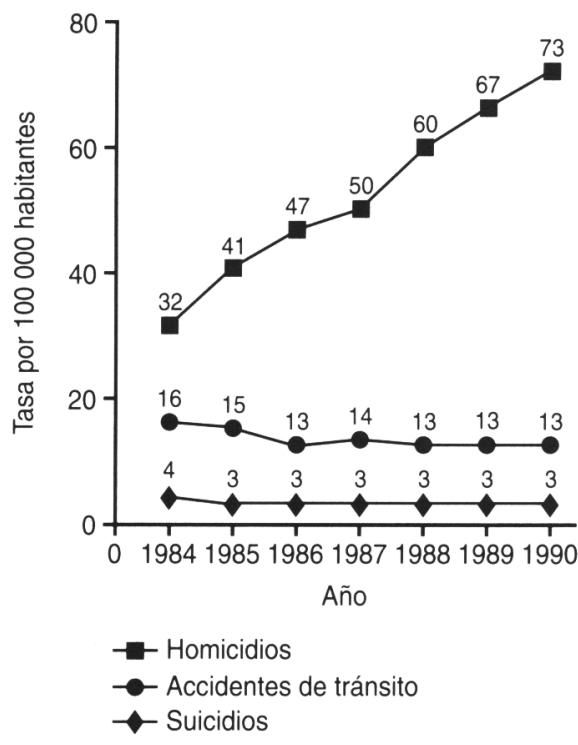

Fuente: Véase la referencia 10.

la vida y la integridad personal serían cometidos en áreas urbanas (26). Es innegable que la violencia tiende a urbanizarse, pero ello no significa la extinción de la violencia rural. Por

\section{FIGURA 2. Homicidios, por edad y sexo. Colombia, 1994}

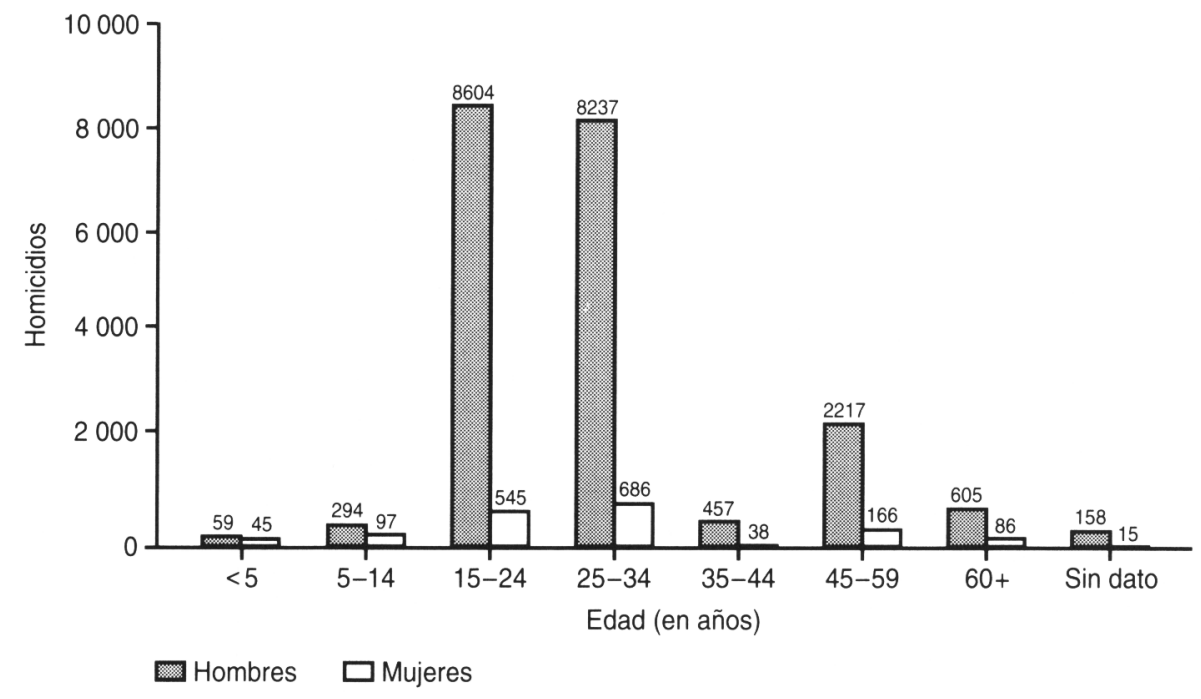

Fuente: Véase la referencia 21 
ejemplo, en el departamento del Guaviare, que es principalmente rural, los homicidios representan $61 \%$ de todas las muertes (23).

La categoría "causas externas" incluye también los accidentes de tránsito, por una lógica que es debatible. Si bien una parte indeterminada de estos accidentes refleja el tipo de violencia ya descrito, otra parte difícil de cuantificar cabe rigurosamente dentro de la categoría de fenómenos accidentales, la cual excluye la intencionalidad y la premeditación. Al desagregar las "causas externas" de muerte en Colombia en sus tres componentes principales -homicidios, accidentes de tránsito y suicidios- y observar sus tendencias en 7 años consecutivos, se confirma que los homicidios han aumentado notablemente, mientras que los accidentes de tránsito y suicidios se han mantenido en niveles bajos y casi uniformes (figura 1).

En 1984 se registraban dos homicidios por cada muerte por accidente de tránsito y ocho homicidios por cada suicidio. Diez años después, en 1994, los homicidios eran cuatro veces más frecuentes que los suicidios y que las muertes en accidentes de tránsito, según las cifras del INMLCF. Las personas entre los 14 y los 34 años de edad representan $60 \%$ de los suicidios, observándose una razón de cuatro hombres por cada mujer que se suicida. En el departamento de Antioquia, donde los homicidios tienen una marcada tendencia ascendente desde 1984, las muertes por accidentes automovilísticos muestran una tendencia descendente sostenida desde 1985, mientras que los suicidios se han mantenido uniformemente bajos (27).

Hay muchos datos y algunos estudios sobre la distribución de las víctimas de la violencia en Colombia según su estrato socioeconómico. Un trabajo del Ministerio de Salud publicado en 1993 (28) reveló que las muertes por accidentes de tránsito tienen una distribución casi uniforme en todos los estratos, mientras que la frecuencia del suicidio aumenta progresivamente a medida que sube el estrato, y la del homicidio a medida que este baja.
La magnitud del problema de la violencia en Colombia se puede apreciar mejor cuando se hacen comparaciones con otros países. En las Américas, por ejemplo, Colombia ocupa el primer lugar en términos de la mortalidad por todo el conjunto de causas de violencia. Según cifras de la OPS, en 1990 la tasa correspondiente era de 76 por 100000 habitantes, y el segundo lugar lo ocupaba Puerto Rico, con una tasa de 27 por 100000 habitantes. Las cifras de homicidos eran aun más alarmantes. Según la misma fuente, en 1990 Colombia encabezó la lista de las Américas con una tasa de 73 homicidios por 100000 habitantes, mientras que la tasa más baja la tuvo Canadá, con 2 homicidios por 100000 habitantes. En cambio en accidentes y suicidios el país ocupó posiciones intermedias. En 1990 Canadá registró la mayor tasa de suicidios, con 13 por 100000 habitantes, mientras que Colombia tuvo una tasa de 3 por 100000 habitantes. En 1989, Chile registró la mayor tasa de accidentes -66 por 100000 habitantes-, mientras que el año siguiente Colombia tuvo solo 39 accidentes por 100000 habitantes. En conjunto, Colombia representa aproximadamente $15 \%$ de todas las muertes violentas y $20 \%$ de todos los homicidios en América Latina y el Caribe. Si se comparan los homicidios en Colombia y Estados Unidos, el contraste es alarmante. De 1984 a 1989, este último país tuvo una tasa máxima de homicidios en la población de 10 a 24 años de 12 por 100000 habitantes, mientras que la tasa colombiana ascendió a 63 por 100000 habitantes en 1989 y a 75 por 100000 habitantes en 1990 (figura 3). La diferencia fue aun más marcada en el grupo de 20 a 24 años. En 1989 Estados Unidos tuvo una tasa de 19 homicidios por 100000 habitantes - tasa muy superior a la aceptable para los países que se consideran desarrollados-, mientras que ese mismo año Colombia tuvo 126 por 100000 habitantes. En otras palabras, ese año la tasa colombiana fue seis veces mayor que la de Estados Unidos.

En vista de todo lo anterior, no se exagera al decir que Colombia padece
FIGURA 3. Tasas de mortalidad (por 100000 habitantes) por homicidios en la población de 10 a 24 años de edad. Colombia y Estados Unidos de América, 1984 a 1990

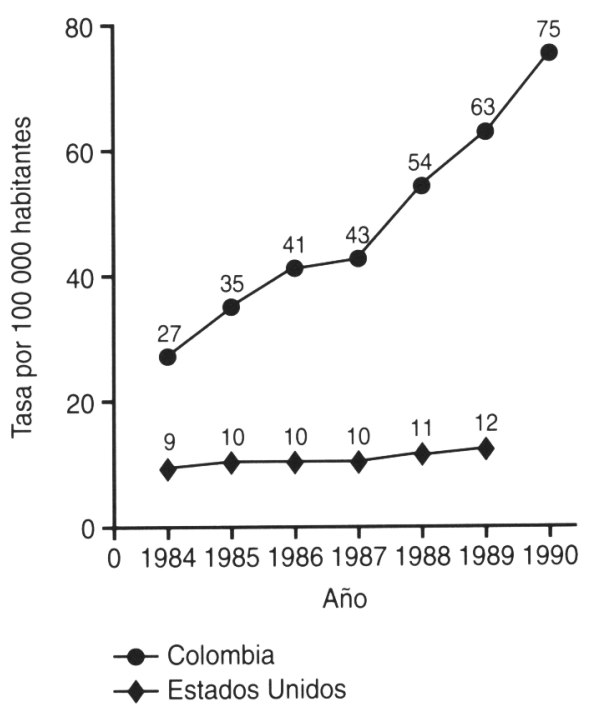

Fuente: Véase la referencia 10

una verdadera epidemia de homicidios. Más bien, parece un reconocimiento tardío de un aspecto de la realidad nacional. La violencia no es solo una epidemia, sino que actualmente constituye el principal problema de salud pública del país, tanto en términos de mortalidad, como de dolor, enfermedad, deterioro de la calidad de la vida y costos económicos para la sociedad y para el sector de la salud, como se verá más adelante. Resulta obvia la importancia del sida y es comprensible la preocupación por su propagación, pero desde que se describió la enfermedad hace más de una década, todavía no ha llegado a 10000 el número de colombianos que han muerto por esa causa. En un período más breve ha habido, como se anotó anteriormente, más de 230000 colombianos asesinados. Con todo, lo importante no es la denominación epidemiológica de la violencia ni el consenso en la asignación de prioridades, sino el reconocimiento del problema, la decisión política para enfrentarlo y la conversión de esa decisión en acciones sostenidas. 


\section{Carácter cotidiano de la violencia en Colombia}

La violencia como mecanismo para resolver conflictos ha penetrado profundamente en los diversos estratos, escenarios y relaciones de la sociedad colombiana. $\mathrm{Su}$ persistencia e intensidad le han otorgado cierta semblanza de normalidad. Considero, incluso, que estamos empezando a vivir dentro de un orden violento (5), es decir, que en la sociedad colombiana se están imponiendo formas de relación caracterizadas esencialmente por mecanismos de fuerza, coacción y miedo. No se pretende insinuar que solo en Colombia se registren hechos violentos, o que sea la violencia la única forma de interacción entre personas, individuos e instituciones, o entre ciudadanos y el Estado. Se trata, más bien, del predominio y aceptación de las acciones violentas sobre todas las demás formas posibles de relación, y del casi inevitable contacto cotidiano, en las más íntimas vivencias individuales y colectivas, con las consecuencias de la violencia. Lo anterior se observa a diario tanto en el hogar y el trabajo, como en la calle, la escuela, el estadio deportivo y la casa.

Según datos del INMLCF y de organizaciones que luchan contra el secuestro, durante 1994 se registró un promedio diario de 73 homicidios, 4 suicidios y 4 secuestros en el país (21). Pero cada día acontecen, además, centenares de casos de violencia intrafamiliar y decenas de delitos sexuales, y se violan otros derechos humanos, como el derecho al trabajo, a la salud y al estudio. Las más mínimas diferencias por conflictos de intereses, opiniones y derechos, o por gustos y amores, se tratan de resolver por la vía contundente de la fuerza. Bien sea por una transgresión en las normas del tránsito vehicular; una derrota o un triunfo deportivo; una disputa entre partes limítrofes; una pequeña deuda, o un proceso judicial, la violencia suele ser la opción más inmediata y definitiva.

De las muchas formas de violencia cotidianas, algunas han venido recibiendo especial atención. Una de ellas es la intrafamiliar, sobre la cual se han celebrado reuniones y se ha publicado recientemente una compilación de investigaciones, y contra la cual se han creado mecanismos de respuesta, tales como las Redes de Prevención (29-31). Huelga decir que ya no es posible hablar de la familia como unidad integrada por padre, madre, hijos y otros parientes. Hoy en día la familia tiene un perfil más amplio y puede caracterizarse mejor como un conjunto de relaciones mediadas por lazos consanguíneos y cuyas formas son múltiples y cambiantes. Como escenario cotidiano de afectos, poder, pasiones, intereses y diferencias, es campo potencial de violencia. Debido al carácter cotidiano y complejo de las relaciones familiares, y a la dualidad entre la vida pública y privada, existen pocas dudas de que los datos registrados sobre la violencia intrafamiliar representan apenas un porcentaje bajo y una imagen parcial de lo que en realidad acontece en el seno de la familia. Aun así, y a pesar de que el INMLCF solo recibe los casos de mayor gravedad con implicaciones penales, dicha entidad atendió durante 1994 un promedio diario de 93 casos de violencia intrafamiliar, de los cuales $75 \%$ se relacionaron con problemas conyugales y $13 \%$ con maltrato infantil. De cada 21 víctimas de maltrato conyugal, 20 son mujeres (21). Según una encuesta en Colombia (32), una de cada tres mujeres que conviven con un compañero ha sido insultada, una de cada cinco ha sido golpeada y una de cada 10 ha sido violada sexualmente. De acuerdo al estudio ya citado del Ministerio de Salud (28), el maltrato verbal contra las mujeres es frecuente en todos los estratos sociales, mientras que el físico parece más frecuente en los estratos bajos.

El maltrato infantil merece especial atención. Ya se señaló, por un lado, la creciente mortalidad por violencia entre los niños. En el primer semestre de $1994,6 \%$ de los homicidios cometidos en Medellín se registraron en menores de 15 años. De los 18 homicidios que en promedio se cometen cada día en Santa Fe de Bogotá, cuatro son en niños, lo cual equivale a un promedio de un homicidio infantil cada 6 horas.

Según una encuesta del Instituto Colombiano de Bienestar Familiar, la cuarta parte de los niños bogotanos han sido maltratados por sus padres. Tanto el maltrato físico como el verbal parecen más frecuentes en los estratos socioeconómicos medios y bajos y las niñas suelen ser las principales víctimas de la violencia sexual registrada. El INMLCF emitió durante 1994 un promedio diario de 30 dictámenes por delitos sexuales. En el primer trimestre de 1995 este número superó en 8\% al del primer trimestre del año anterior. De todos los dictámenes, $60 \%$ correspondieron a casos de violencia contra menores de 14 años, observándose una relación de 10 mujeres por cada varón (33).

Existen otras formas frecuentes de maltrato infantil, como el abandono, la explotación laboral y la marginación. También constituyen formas de violencia generalizada contra los niños los desplazamientos forzosos, con sus secuelas de desarraigo cultural, desintegración familiar y desajuste económico, y la orfandad (34).

Los niños y niñas no siempre son las víctimas de las redes de violencia. Pueden ser también -lo son con creciente frecuencia- los agresores. En 1993 se abrieron 14461 nuevos expedientes judiciales contra niños infractores, cifra que en 1995 superó los 35 000. Las filas de la guerrilla, del sicariato y de la delincuencia organizada y no organizada se engrosan progresivamente con niños cada vez menores. Su participación varía desde tareas de apoyo y complicidad, hasta responsabilidades directas en la ejecución de delitos y homicidios. Hay información fehaciente de la participación de niños en la ejecución de las recientes masacres de Urabá. El tema reviste especial interés y es motivo de gran desasosiego, particularmente cuando se consideran sus implicaciones para el futuro. Con él se relacionan muchos otros temas: la crisis de la estructura familiar; la violencia vivida en la infancia y su reproducción en el adulto; la falta 
de equidad; los contenidos y métodos de la educación formal e informal; el papel de los medios de comunicación en la difusión de información y en la creación de conciencia y valores sociales; los fundamentos éticos; la penalización y rehabilitación de niños delincuentes. Más que un interés analítico, el problema debe provocar una reacción social, si realmente se desea sentar las bases para un futuro menos violento, tanto en los ámbitos político y social como en la vida cotidiana.

Su presencia diaria también redunda en una especie de banalización de la violencia, es decir, en su aceptación como fenómeno intrascendental. Aumentan los niveles de tolerancia frente a ella, se reducen los mecanismos de respuesta individual y colectiva, se pierde la capacidad de asombro y se sucumbe ante el miedo y la indiferencia. Esta banalización añade un nuevo eslabón al problema y conduce a cierto fatalismo, de perversas consecuencias en el momento de intentar la activación de mecanismos de reacción.

\section{El sicariato, los desechables y las masacres}

Estos tres fenómenos forman parte cotidiana de la violencia en Colombia y son altamente representativos de la actual situación de violencia. Ninguno es un invento ni un patrimonio exclusivamente nacional, pero en la última década los tres han cobrado mayor presencia e importancia en el país. Una breve reflexión sobre ellos, como la que sigue, permite reconocer lo que representa actualmente la violencia, advertir la gravedad de su persistencia y hacer un llamado a combatirla. Es imposible considerar sano a un país o sociedad que genera masacres, alimenta el sicariato y produce y elimina "desechables".

La palabra "desechable" viene del mundo industrial. Se refiere originalmente a productos y objetos que deben eliminarse cuando termina su vida útil. El concepto se humanizó a partir de la configuración de lo que Hanna Arendt denominó hace décadas "poblaciones superfluas", cuyo perfil vienen explorando en el Brasil algunos investigadores (35). En Colombia la categoría no se refiere a un grupo homogéneo, sino a grupos de diferente naturaleza, pero unificados por una misma valoración: sobran, son indeseables e infuncionales $y$, por tanto, deben ser eliminados. Desechable es para unos el que asume una conducta sexual diferente, como la prostituta, el travesti, el homosexual; para otros, es el ladrón callejero, el opositor político, el rival en el amor o en el mercado, el mendigo, el minusválido, el de otra raza. Frente a su exterminio los sectores sociales que los califican como tales no solo adoptan una postura permisiva, sino promotora o activa. Si en el orden violento matar es la forma habitual de intentar resolver un conflicto, matar al desechable es una tarea de "limpieza social", concepto que se ha prestado para justificar el exterminio de muchos de ellos.

Más que los sicarios, el verdadero problema es el sicariato. Los primeros son los asesinos a sueldo, las terminales de circuitos complejos, la cara externa de actores ocultos. Es grave que existan sicarios, pero lo es más aún que exista el sicariato; es grave que alguien mate porque le pagan, pero es más grave que alguien pague por matar. Ambos fenómenos tienen una misma fundamentación: es válido asignar y pagar o recibir un precio en dinero por la eliminación del derecho a la vida de otro. Pero mientras el sicario ejecuta sin más objetivo que la remuneración, los verdaderos protagonistas del sicariato son quienes, en función de sus valores e intereses, seleccionan a la víctima, le ponen precio, proveen el dinero y contratan la acción. Todo este proceso sicarial niega categóricamente cualquier ordenamiento jurídico, el carácter innegociable de la vida humana, algunos de los papeles esenciales asignados al Estado y las relaciones de ciudadanía.

Llama la atención que en Colombia, país en que el fenómeno del sicariato ha alcanzado proporciones alarmantes, se haya avanzado tan poco en la comprensión de sus raíces, en la desar- ticulación de sus redes y en la identificación de sus representantes. Quizá haya contribuido a ello su vinculación con el complejo y peligroso fenómeno del narcotráfico. Pero, para superar los actuales niveles de violencia se requiere, entre muchas otras cosas, comprender el fenómeno del sicariato $\mathrm{y}$ proceder a combatirlo.

Las masacres son asesinatos colectivos y simultáneos. Se vienen registrando en el país con mayor frecuencia desde la década pasada y se concentran por temporadas en determinadas zonas. Según los principales periódicos del país, en los primeros 10 meses de 1994 se registraron en el valle de Aburrá, donde se ubica la ciudad de Medellín, 43 masacres con un total de 179 víctimas y un promedio de cuatro muertos por masacre, la mayoría hombres jóvenes. En agosto y septiembre de 1995, las masacres se concentraron en la zona bananera de Urabá, donde hubo ocho masacres con un total de 91 muertos y un promedio de 11 muertos por episodio. En el municipio de Apartadó de la misma zona, se cometió en enero de 1994 una de las mayores masacres en la historia reciente del país: 36 muertos y 17 heridos, todos residentes de un barrio de invasión y muchos de ellos reintegrados a la vida civil después de haber pertenecido a un grupo guerrillero.

Las masacres no siempre apuntan hacia el mismo blanco. Algunas se dirigen a eliminar a un tipo de desechable en particular, otras a cobrar cuentas pendientes entre traficantes de drogas o de armas, a eliminar opositores o rivales políticos, a ostentar fuerza y dominio en determinados territorios y a infundir miedo por atreverse a disentir. Incluso hay indicios fundados de que algunas masacres han sido la prueba final del entrenamiento para optar por el título de sicario. En todos los casos, la colectivización de la muerte tiene un efecto simbólico y de intimidación. Las masacres reflejan la vulnerabilidad de la sociedad, el predominio de los intereses de personas o grupos por encima de cualquier pauta o concepto elemental de convivencia, la pérdida del monopolio de la fuerza 
por parte del Estado y, especialmente, el actual grado de desvalorización de la vida humana. Además, por su propia dinámica, más de una masacre ha sido una respuesta a otra anterior, generándose una especie de círculo perverso de asesinatos "en masa". Las masacres, como hechos de violencia colectiva, demandan acciones y reacciones también colectivas que les resten legitimidad y que ayuden a controlar o eliminar los factores que las fomentan.

\section{COSTOS DE LA VIOLENCIA PARA EL SECTOR DE LA SALUD}

Bien sea desde una perspectiva económica, social, emocional o psicológica, son muy altos los costos de la violencia para el sector de la salud en Colombia. En este documento se incluyen en la categoría de costos tanto los bienes, recursos y fondos que se dedican a mantener los servicios de atención y recuperación, o que se pierden a causa de los daños sufridos, como el impacto negativo sobre la calidad de la vida individual y colectiva. El tema obviamente exige una mayor precisión conceptual e investigaciones directas y de mayor profundidad. Para los objetivos del presente trabajo basta con señalar algunos indicadores que ilustren con precisión el costo casi insospechado para la salud y para el sistema sanitario del país de las actuales condiciones de violencia.

Varios grupos y autores, aplicando muy diversas metodologías, han estudiado aspectos del tema en distintos países o subregiones (36-41). Se trata, por lo general, de estudios locales, sectoriales o regionales cuyas metodologías aún no permiten calcular los costos globales de la violencia ni conocer a cabalidad muchos de los aspectos que aquí se consideran importantes. Los indicadores más utilizados han sido las ya mencionadas tasas de morbilidad y mortalidad por causas externas; los años de vida potencial perdidos; el porcentaje de la mortalidad atribuible a causas externas; el porcentaje de camas hospitalarias y de días de estancia hospitalaria dedicados a víctimas de la violencia; y los costos directos de la atención médica y hospitalaria ocasionados por ella. Recientemente algunos investigadores e instituciones han venido empleando el indicador denominado "años de vida saludable perdidos", que equivale a los años perdidos no solo por muerte prematura sino también por discapacidad. Donde se dispone de menos información es quizá en el campo de los indicadores del deterioro de la calidad de vida por causa específica, y este campo es, por lo tanto, el que exige un mayor trabajo futuro.

De los estudios citados, uno de los más interesantes, ya que incluye datos parciales de Brasil, México y Perú, es el patrocinado por la División de Promoción y Protección de la Salud de la OPS (41). Con todo, se trata de estudios de caso que solo exploran los costos de la atención hospitalaria y que se basan en metodologías, universos y períodos diferentes. En México y Perú, por ejemplo, se usaron como referencia los datos reunidos en un solo servicio dentro de un solo hospital mediante una encuesta que duró una semana. No obstante, los autores realizaron análisis prospectivos para todos los países de América Latina y el Caribe y concluyeron que el costo de la atención prestada a $50 \%$ de las víctimas que murieron y a $100 \%$ de las que sufrieron lesiones de leves a graves fluctuaba en la Región entre US\$ 3600 millones y US\$ 5600 millones, cifra que representa de 4 a $7 \%$ del gasto total en salud de todos los países en conjunto. En estos mismos países, las pérdidas económicas ocasionadas por las muertes prematuras y discapacidades producidas por la violencia se estimaron en US\$ 11400 millones.

En Colombia los homicidios y accidentes son la causa principal de años de vida potencial perdidos. En 1991, dicha categoría representó 39,9\% del total de años de vida potencial perdidos, y las causas perinatales ocuparon el segundo lugar, con $13,3 \%$ (42). Según la misma fuente, en la década de 1983 y 1992, la proporción de años de vida potencial perdidos por causas relacionadas con la violencia y los accidentes mostró una tendencia ascendente. Si se parte de la premisa de que la actual esperanza de vida al nacer de un colombiano promedio es de 69 años, y de que la edad promedio de las víctimas del homicidio es de 29 años, se deduce que por cada asesinato se pierden en promedio 40 años de vida potencial. Si se estima en aproximadamente 30000 el número anual de homicidios, la deducción es que anualmente el país pierde por esta causa 1200000 años de vida potencial. Mientras que en 1983 la violencia y los accidentes representaban $17 \%$ de toda la mortalidad en el país, en 1991 dicho porcentaje se elevó a $27 \%$. Esto implica que de cada cuatro colombianos muertos, uno muere por violencia o accidente. En Antioquia, donde se produce un alto porcentaje de los homicidios, se calculó en 64833 el número de años de vida potencial perdidos por dicha causa en 1982 , cifra que equivale a $20 \%$ del total de años de vida potencial perdidos. En 1992, la cifra absoluta había subido a 507200 y la fracción correspondiente a la escandalosa cifra de $84 \%$ (27). En cuanto a la denominada "carga de la enfermedad", estimada en términos de años de vida saludable perdidos, los homicidios ocupan el primer lugar. En el período de 1989 a 1991, fueron los responsables de 1356675 años de vida saludable perdidos, o $24,6 \%$ del total, porcentaje que duplica el atribuible a las enfermedades cardiovasculares (43). Si no fuera por la violencia, la esperanza de vida promedio de los colombianos sería mayor de la actual cifra de 69 años. Esto queda confirmado por el hecho, ampliamente comprobado, de que la esperanza de vida de las mujeres es mayor que la de los hombres y de que la brecha entre los sexos muestra una tendencia a incrementarse.

En lo referente a los costos monetarios, las cifras aún son incompletas, poco confiables, o demasiado generales y se basan en unidades que siguen siendo objeto de debate. Una publicación económica nacional (44), basada en un estudio del Departamento Nacional de Planeación, divulgó 
recientemente algunas cifras que reflejan el costo total de la violencia en Colombia. Según la publicación, cada año el país gasta por concepto de violencia \$Col 6200 millones. El total se obtuvo sumando los gastos en defensa, seguridad pública y privada, justicia, pagos por secuestros, costos por delitos contra el patrimonio público y privado y valor estimado de los homicidios en términos de años de vida potencial perdidos. No aparece la estimación de los gastos del sector de la salud. Aunque la cifra arroja luz sobre la magnitud del problema, es imprecisa porque incluye rubros demasiado amplios y excluye otros que son esenciales.

En 1993, el entonces Ministro de Salud estimó en \$Col 600000 (US\$1,00 $\approx \$$ Col 1000,00 ) el costo promedio de la atención de un caso de trauma y en \$Col 80000 millones el costo anual de la atención de casos de violencia y trauma en el país. Ese mismo año, el Director del Hospital Universitario San Vicente de Paúl de Medellín, entidad que dedica la cuarta parte de sus camas a la atención de casos graves de violencia y en la cual la tercera parte de los egresos son de casos atendidos por lesiones personales, estimó en \$Col 5340 millones el costo de la atención del trauma en la institución, equivalente a un promedio diario de \$Col 15 millones. En 1990 la tercera parte del presupuesto total de dicho hospital se dedicó a cubrir los costos de hospitalización por lesiones personales (39). En 1994 el mismo hospital recibió en su servicio de urgencias de adultos un promedio mensual de 1268 casos de trauma y violencia. Si se mantiene el mismo promedio estimado de \$Col 600000 por la atención de cada caso, el costo mensual aproximado en la institución será de \$Col 760 millones y el anual de \$Col 9120 millones. ¿Quién paga estas cantidades, proyectadas ya a nivel nacional?

Otros costos que no se han calculado con precisión son los de las secuelas orgánicas, psíquicas y físicas de los actos de violencia que no causan la muerte, y los de otras consecuencias indirectas. Son ejemplos la pérdida o limitación de las extremidades u órganos, las alteraciones del desarrollo y del funcionamiento psicológico, afectivo, intelectual y motor, y la disminución de la agudeza sensorial y perceptiva. Tampoco son menos cuantiosos los costos de la rehabilitación de las funciones recuperables, ni los de los servicios de medicina legal y forense, esenciales para la toma de decisiones jurídicas y penales y para el avance del trabajo científico y técnico en el campo medicolegal.

Es posible que los costos mayores de la violencia no se puedan estimar en términos cuantitativos o económicos. ¿Quién puede estimar el costo de quedar huérfano o de perder al cónyuge? ¿Cómo valorar el impacto de una masacre en los parientes, vecinos, compañeros y amigos de las víctimas, y en la población en general? ¿A cuántas unidades de dolor e infelicidad constantes equivale tener para siempre a un familiar o amigo desaparecido? ¿Y cómo estimar, en términos del bienestar de individuos y grupos, el costo de los desplazamientos y exilios forzados por las amenazas y otras formas de violencia? No se puede disfrutar, ni dormir, trabajar o comer de la misma manera tras el asesinato o la desaparición de un hijo, pariente o amigo. La salud mental es una de las cajas de resonancia interior de la violencia padecida directa o indirectamente, como lo son también la integridad del tracto gastrointestinal y del cerebro y la presión arterial. Es posible que la dosis diaria de risa de los colombianos haya disminuido notablemente en la última década, en buena parte debido a las múltiples violencias padecidas. Y hay indicios, que deben comprobarse rigurosamente, de que han aumentado las consultas y el consumo de medicamentos por depresión, gastritis, cefalea e insomnio.

\section{RESPUESTA DEL SECTOR DE LA SALUD A LA VIOLENCIA}

No se suele dar reconocimiento al sector de la salud en su conjunto, ni a cada uno de sus miembros en particu- lar, por su cotidiana respuesta, con limitaciones de recursos en ocasiones extremas, a las demandas que diariamente plantea la atención a las víctimas de la violencia. Hay muchas historias no contadas de genialidad, solidaridad y heroísmo en este campo. El objeto de estas reflexiones finales es identificar los diferentes frentes en que el sector de la salud puede, junto con otros sectores, contribuir a mitigar el problema de la violencia y a encontrar alternativas para lograr una sana convivencia ciudadana. Nuestra discusión se basará, por consiguiente, en la premisa de que la respuesta de las diferentes instituciones del sector de la salud de Colombia frente a la violencia es aún insuficiente e inadecuada, si se consideran la magnitud, dinámica y progresión del problema.

Cabe, para empezar, hacer dos observaciones críticas. En primer lugar, el sector de la salud, en vez de contribuir a enfrentar y atenuar la violencia, muchas veces genera más violencia. Estructuralmente, la falta de cobertura sanitaria de casi la tercera parte de la población colombiana es, de por sí, una forma de violencia importante. Además, cuando el sector, por intermedio de alguno de sus agentes, impone autoritariamente sus pautas y decisiones o pretende obviar elementos importantes de la subjetividad del paciente, puede incurrir en abiertas violaciones de los derechos humanos y en consiguientes actos de violencia. Ejemplos frecuentes son la práctica de ciertos procedimientos en mujeres sexualmente violadas o el ingreso involuntario del paciente.

En segundo lugar, generalmente nos quedamos cortos frente al problema de la violencia, es decir, la base conceptual, los mecanismos de ejercicio profesional y la asimilación y apropiación de determinadas funciones sociales, nos llevan con frecuencia a reducir la práctica y el saber médicos a parcelas pequeñas de la realidad, que en este caso sería la violencia. Por tal motivo terminamos por desconocer la índole, magnitud y dinámica del problema en cuestión, lo cual empobrece el aporte potencial del sector de la salud y con- 
tribuye a fraccionar el conocimiento y enfrentamiento del problema.

En principio pueden distinguirse tres tipos de instituciones del sector de la salud que obligatoriamente guardan relación con la violencia y de las cuales la sociedad tiene derecho a exigir una mejor respuesta y mayor colaboración. Se trata de las instituciones formadoras de personal; las encargadas de atender y rehabilitar a las víctimas; y las responsables de las actividades de orientación, gestión, financiamiento y evaluación.

Si se examina el contenido temático de los planes de estudio de técnicos, auxiliares, profesionales y otros especialistas en el campo de la salud, así como las áreas de práctica, líneas de investigación, habilidades cultivadas y horas dedicadas al tema de la violencia, se advierte un enorme desajuste entre la magnitud de este problema en Colombia y la importancia que se le otorga. Ya es hora de lograr una integración más orgánica, fundamentada y permanente de los distintos aspectos del tema en las diferentes etapas del proceso formativo. Si la magnitud social del problema se ha de ver reflejada en los intereses, contenidos y actividad diaria de la academia, la violencia como objeto de conocimiento y de transformación, y su enfrentamiento como responsabilidad social, tendrían que figurar mucho más entre los temas del ciclo formativo y suscitar un mayor esfuerzo intelectual y mayor creatividad en el diseño de estrategias, mecanismos y acciones de respuesta.

En lo que respecta al segundo punto planteado anteriormente, las escuelas deben cuidarse de "medicalizar" la violencia, es decir, de reducirla a la lógica bionatural y de pretender enfrentarla con los recursos y disciplinas tradicionalmente utilizados por las diferentes profesiones de la salud. Por su naturaleza, dinámica, significados e implicaciones, comprender y manejar la violencia requiere un esfuerzo intersectorial, interdisciplinario, multiprofesional y participatorio. Hay que superar la ignorancia y los prejuicios en las áreas de economía y derecho, sociología y ética, historia y filosofía. Hay que asomarse a otros escenarios fuera de los consultorios y hospitales, escuchar a interlocutores diferentes de los pacientes, asociarse con nuevos actores y crear nuevos frentes de trabajo.

Como parte de la comunidad académica nacional, los investigadores han desempeñado un papel importante en el esclarecimiento del problema de la violencia en Colombia. No obstante, tan grande es el desajuste entre las necesidades sociales y las prioridades científicas, que queda aún mucho camino por recorrer. Tanto la epidemiología como la medicina legal y las ciencias forenses; la vigilancia epidemiológica como los más modernos sistemas de información; los conocimientos sobre las bases sociales, médicas y fisiopatológicas de la violencia y del trauma, podrían y deberían tener en Colombia un gran desarrollo científico. En la comprensión científica del problema y en la creación de conciencia social frente a él, la academia tiene ante el país una tarea inconclusa.

Los servicios asistenciales han pagado una cuota muy alta por atender a quienes sufren las peores consecuencias de la violencia. Son sus trabajadores los que han soportado la presión de las crisis y desesperación de las víctimas y sus familias. Las instituciones han tenido que multiplicar sus recursos, generalmente escasos, para satisfacer una enorme demanda.

Es preciso, no obstante, reconocer también que con frecuencia la respuesta ha sido pasiva y los recursos insuficientes en relación a la naturaleza, dinámica y magnitud de la violencia. Formado el personal sin suficiente visión y capacitación para entender el problema y enfrentarlo, diseñados los servicios para determinadas formas $\mathrm{y}$ tipos de atención, y limitados estructuralmente por graves carencias, es aún precaria la adecuación del sector sanitario a las necesidades impuestas por la violencia. Ella exige comprensión psicológica; un equipo multiprofesional de respuesta rápida y trabajo coordinado en red; mecanismos ágiles de intervención y remisión; materiales, equipos e instalaciones adecuadas; una experiencia asistencial sistematizada y mecanismos permanentes de evaluación y ajuste. Pero además, las instituciones deberían dedicarse directamente, sin sacrificar su responsabilidad asistencial, a apoyar e intensificar las acciones preventivas, a promover la salud y a defender la vida. Estas actividades requieren la acción de múltiples sectores, recursos adicionales y la capacidad de entablar nuevas relaciones y de correr nuevos riesgos. Obviamente los servicios asistenciales no pueden solucionar el problema, pero sí pueden desempeñar un papel importante a la hora de combatir uno de sus aspectos más fundamentales. Tales servicios contribuirían en mucho mayor grado a atenuar el impacto de la violencia - vidas perdidas, dolor humano, costos monetarios, y demás-si en su orientación, estructuración, funcionamiento y financiamiento la violencia ocupara el lugar que merece en proporción con su magnitud.

Algo similar a lo anterior puede afirmarse en relación con las entidades e instituciones dedicadas a la formulación de políticas, a la toma de decisiones y al financiamiento, programación, gestión y evaluación del sector de la salud. No se pretende reestructurarlo todo en función de la violencia, pero resultan contraproducentes y muy costosas la ignorancia y subvaloración del problema. No puede tratarse de forma marginal o sintomática $\mathrm{o}$, peor aún, por oportunismo político, un problema que responde por la cuarta parte de las muertes y la tercera parte de los servicios que presta el sistema de salud. Conviene reiterar la importancia de la cooperación internacional, de abordar el problema de una manera intersectorial e interinstitucional, y de articular las políticas y planes nacionales con los de otros países para potenciar las iniciativas y recursos.

En un reciente documento propuse articular la labor científica y política de la comunidad medicosocial internacional para construir y poner en práctica una agenda en pro de la vida (45). Si hoy en día la violencia constituye en Colombia la principal amenaza a la vida y al bienestar, con mayor razón es preciso, para combatirla, ir más allá de 
las acciones e intervenciones particulares y sectoriales y articular todo el trabajo de los sectores de la salud pública y epidemiología, procurando lograr una movilización a gran escala en defensa del derecho a la vida y la salud. Ello implica tener políticas y opciones generales bien definidas en los niveles individual y colectivo; llegar a una serie de acuerdos intra e interinstitucionales en los niveles local, departamental y nacional; dar prioridad a ciertos aspectos, tareas, recursos y mecanismos, y conseguir el apoyo activo de los medios de comunicación. Además de su poder de convocatoria y de su capacidad de acabar con la apatía y la tolerancia a la violencia, la agenda en pro de la vida y el movimiento en pro de la salud poseen la capacidad de convertirse en mecanismos concretos de participación ciudadana y en vehículos para reformular la acción estatal y fortalecer la sociedad civil. Todos estos son mecanismos esenciales para revertir en un plazo razonable la intolerable espiral de violencia que hoy registra y padece Colombia.

\section{REFERENCIAS}

1. Guzmán CG, Umaña LE y Fals-Borda O. La violencia en Colombia: monografías sociológicas. Santa Fe de Bogotá: Universidad Nacional; 1962.

2. Bejarano J, Fals-Borda O, Fajardo D, et al. Once ensayos sobre La Violencia. Santa Fe de Bogotá: Centro de Estudios de la Realidad Colombiana, Centro Gaitán; 1985.

3. Comisión de Estudios sobre la violencia. Colombia: violencia y democracia. Santa Fe de Bogotá: Universidad Nacional de Colombia, Instituto Colombiano para el Desarrollo de la Ciencia y la Tecnología Francisco José de Caldas; 1989.

4. Franco S. Violencia y salud. Rev Universidad Antioquia 1990;220:18-27.

5. Franco S. Violencia, ciudadanía y salud pública. Santa Fe de Bogotá: Corporación Salud y Desarrollo; 1995. (Serie Documentos Especiales No. 4).

6. Minayo MC. A violencia social sob a perspectiva da saúde pública. Cad Saude Publica 1994;10(supl 1):7-19.

7. Organización Panamericana de la Salud. Violencia y salud: resolución XIX. Washington, DC: OPS; 1993. (Documento inédito).

8. Franco S. La violencia: un problema de salud pública que se agrava en la Región. Bol Epidemiol OPS 1990;11:1-7.

9. Camacho GA. Dimensiones de la democracia y la violencia en las Américas. Washington, DC: Organización Panamericana de la Salud; 1994. (Documento PAHO/HPP/94.23).

10. Yunes J, Rajs D. Tendencias de la mortalidad por causas violentas en la población general y entre los adolescentes y jóvenes de la región de las Américas. Cad Saude Publica 1994; 10(supl. 1):88-125.

11. Fals-Borda O. Lo sacro y lo violento: aspectos problemáticos del desarrollo en Colombia. En: Bejarano J, Fals-Borda O, Fajardo D, et al. Once ensayos sobre La Violencia. Santa Fe de Bogotá: Centro de Estudios de la Realidad Colombiana, Centro Gaitán; 1985.

12. Perea R CM. Porque la sangre es espíritu: imaginario y discurso político en las élites capita- linas. Santa Fe de Bogotá: Instituto Colombiano de Bienestar Familiar; 1995. (En prensa).

13. Restrepo LC. Democracia vivencial y cultura de la convivencia. Rev NOMADAS (Santa Fe de Bogotá) 1995;2:60-67.

14. Salazar, Alonso. No nacimos pa'semilla. Santa Fe de Bogotá: Centro de Investigación y Educación Popular, Corporación Región; 1990.

15. Camacho G A. La Violencia en Colombia: elementos para su interpretación. Rev Foro (Santa Fe de Bogotá) 1988;6:3-12.

16. Camacho GA. Cinco tesis sobre narcotráfico y violencia en Colombia. Rev Foro (Santa Fe de Bogotá) 1991;15:65-73. 1991.

17. Deas M y Gaitán D F. Dos ensayos especulativos sobre la violencia en Colombia. Santa Fe de Bogotá: Tercer Mundo Editores; 1995.

18. De Roux F. Fundamentos para una ética ciudadana. En: Colombia: una casa para todos; debate ético. Santa Fe de Bogotá: Programa por la Paz, Compañía de Jesús; 1991.

19. Kalmanovitz S. Economía de la violencia. Rev Foro (Santa Fe de Bogotá) 1988;6:13-24.

20. Richani I. La economía política de la violencia: el sistema de guerra en Colombia. Santa Fe de Bogotá: Instituto de Estudios Políticos y Relaciones Internacionales; 1995. (Documento mimeografiado).

21. Centro de Referencia Nacional sobre Violencia. Reporte del comportamiento de lesiones fatales y no fatales en Colombia, 1994. Santa Fe de Bogotá: Instituto Nacional de Medicina Legal y Ciencias Forenses; 1995.

22. García H, Vélez C. Muerte violenta por homicidio en Medellín en la década del 80. [Tesis de Maestría]. Medellín: Facultad Nacional de Salud Pública; 1992.

23. Arbeláez MP, Ruiz IC. Vigilancia epidemiológica: indicadores y mapeo de riesgo. Santa $\mathrm{Fe}$ de Bogotá: Ministerio de Salud de Colombia y Organización Panamericana de la Salud; 1994.

24. Iglesia denuncia exterminio de la niñez. El Tiempo. Santa Fe de Bogotá, 30 de septiembre, 1995:15A.

25. Colombia, Ministerio de Justicia y del Derecho. La violencia urbana en Colombia: evidencia empírica y propuestas de política. Santa Fe de Bogotá: Misión Siglo XXI; 1994.

26. Colombia, Policía Nacional. Criminalidad 1990: tendencias de la criminalidad, 1958-1991. Santa Fe de Bogotá: Policía Nacional; 1990.

27. Dirección Seccional de Salud de Antioquia, Oficina de Epidemiología. Series Cronológicas de Salud. Medellín: DSS; 1994.

28. Colombia, Ministerio de Salud. Salud mental y consumo de sustancias sicoactivas. Santa Fe de Bogotá: MINSA; 1993.

29. Redes de Prevención de Violencia Intrafamiliar, Subsecretaría de la Mujer, Instituto Colombiano de Bienestar Familiar. Violencia Intrafamiliar: memorias. Medellín: Litoarte; 1993.

30. Servicio Seccional de Salud de Antioquia. Violencia intrafamiliar: cotidianidad oculta. Medellín: SSSA; 1994.

31. Universidad de Antioquia, Subsecretaría para la Mujer, Despacho de la Gobernación de Antioquia. La violencia: un problema de la vida de familia. Medellín: Universidad de Antioquia; 1995.

32. Profamilia. Encuesta de prevalencia, demografía y salud, 1990. Santa Fe de Bogotá: ProfamiliaDHS; junio de 1990.

33. Centro de Referencia Nacional sobre Violencia, Instituto Nacional de Medicina Legal. Delitos sexuales en Colombia, 1994 y primer trimestre de 1995. Boletín del CRNV (Santa Fe de Bogotá) 1995:1:2-3.

34. Gaylin N, Sadlier K, Salas S M. The effects of chronic community violence on Colombian children and their families. Informe final presentado ante la Organización Panamericana de la Salud, Washington, D.C., septiembre de 1994.

35. Cruz-Neto O, Minayo MC. Extermínio: violentação e banalização da vida: $\mathrm{O}$ impacto da violencia social sobre a saúde. Cad Saude Publica 1994;10(supl 1):199-212.

36. Hospital Universitario San Vicente de Paúl. Análisis de las condiciones de rehabilitación en Antioquia: su relación con causas violentas. Medellín: Universidad de Antioquia; 1987. (Documento mimeografiado). 
37. Médici A. As raizes económicas da violencia e seus impactos na saúde. Trabajo presentado ante el Grupo de Trabajo sobre Violencia y Salud en América Latina, Río de Janeiro, 11 a 14 de diciembre de 1989. (Documento mimeografiado).

38. De Mello J, De Mello MH. O impacto da violencia nos serviços de saúde. Trabajo presentado ante el Grupo de Trabajo sobre Violencia y Salud en América Latina, Río de Janeiro, 11 a 14 de diciembre de 1989. (Documento mimeografiado).

39. Cano G E, Castrillón Z MT. Egresos por lesiones personales, 1989. Medellín: Fundación Hospitalaria San Vicente de Paúl; 1990.
40. Mondragon D. Hospital costs of societal violence. Med Care 1991;30:453-460.

41. Organización Panamericana de la Salud. El impacto económico de la violencia sobre las instituciones de salud en países de América Latina y el Caribe: informe preliminar. Washington, DC: OPS; 1994.

42. Colombia, Ministerio de Salud. La salud en Colombia: diez años de información. Santa Fe de Bogotá: MINSA; 1994.

43. Colombia, Ministerio de Salud. La carga de la enfermedad en Colombia. Santa Fe de Bogotá: MINSA; 1994.

44. Ardila Durán E, Pereira Romero E. La industria de la violencia. Portafolio: Semanario de
Economía y Negocios (Santa Fe de Bogotá) 1995;107:14-16.

45. Franco S. La salud al final del milenio. Analisis Politico 1995;24:51-64.

Manuscrito recibido el 18 de diciembre de 1995. Se aceptó en versión revisada el 20 de junio de 1996.

ABSTRACT In Colombia, violence seems uncontrollable. Along with massacres and group killings of astonishing cruelty, there are also kidnappings and disappearances, abuse of children and the elderly, and rape of young adolescents. Every day, without respite,

Violence and health in Colombia Colombians are witnesses or victims of street crimes as well as racial, sexual, and socioeconomic discrimination. Unwillingly, they become agents of aggression in public transport, at home, at school, and at work.

Colombia has the highest rates of mortality from homicide in the world. Apart from the enormous institutional burden that violence imposes on the health services and forensic medicine, it now constitutes the principal public health problem in the country. To confront it, the health sector must develop policies and finance actions, develop innovative ways to train personnel, implement public education processes, and devote more effort and greater creativity to research, which up to now has provided some, but not enough, important answers.

Violence, which is the substitution of force for any type of dialogue, must be considered within the context of life and health. This it not merely an attempt to rationalize violence, much less to substitute words or reflection for action, but rather an attempt to understand it in depth in order to search for alternatives.

With that goal, this article analyzes the subject of violence in Colombia, principally from the perspective of its effect on the health of the citizens and its implications for the health sector. The author fully recognizes the subjectivity and limitations of the views he expresses herein. 